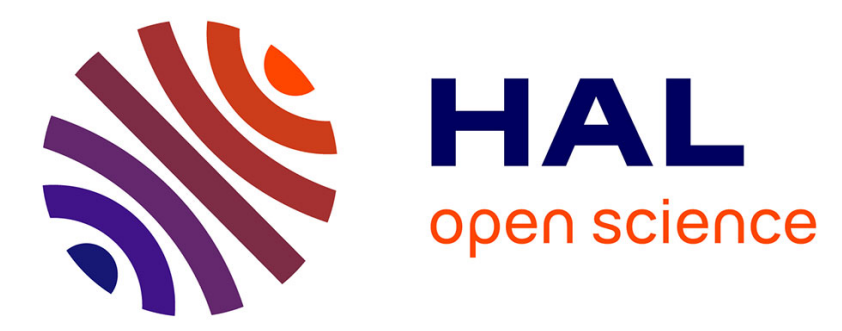

\title{
Étude du spectre d'électrons de conversion de faible énergie émis par le Francium 221 (225Ac $\rightarrow \alpha 221 \mathrm{Fr})$
}

Chin-Fan Leang, Françoise Gautier

\section{To cite this version:}

Chin-Fan Leang, Françoise Gautier. Étude du spectre d'électrons de conversion de faible énergie émis par le Francium 221 (225Ac $\rightarrow \alpha$ 221Fr). Journal de Physique, 1969, 30 (4), pp.296-300. 10.1051/jphys:01969003004029601 。 jpa-00206787

\section{HAL Id: jpa-00206787 https://hal.science/jpa-00206787}

Submitted on 1 Jan 1969

HAL is a multi-disciplinary open access archive for the deposit and dissemination of scientific research documents, whether they are published or not. The documents may come from teaching and research institutions in France or abroad, or from public or private research centers.
L'archive ouverte pluridisciplinaire HAL, est destinée au dépôt et à la diffusion de documents scientifiques de niveau recherche, publiés ou non, émanant des établissements d'enseignement et de recherche français ou étrangers, des laboratoires publics ou privés. 


\title{
ÉTUDE DU SPEGTRE D'ÉLEGTRONS DE GONVERSION DE FAIBLE ÉNERGIE ÉMIS PAR LE FRANGIUM $221\left({ }^{225} \mathrm{Ac} \stackrel{\alpha}{\rightarrow}{ }^{221} \mathrm{Fr}\right)$
}

\author{
Par Chin-Fan LEANG (*) et Françoise GAUTIER,
}

Centre de Spectrométrie Nucléaire et de Spectrométrie de Masse, Bâtiment I04, Campus, 9I-Orsay.

(Reçu le 9 décembre 1968.)

Résumé. - Le spectre d'électrons de conversion émis par ${ }^{221} \mathrm{Fr}$ a été étudié par un spectromètre $\beta \pi \sqrt{ } \overline{2}$. Les multipolarités des transitions intenses ont été déterminées. Un désaccord existe entre $I \gamma$ et $I e^{-}$pour les rayonnements de 73,7 et $99,6 \mathrm{keV}$, ce qui a conduit à l'hypothèse d'un niveau double à $99,6 \mathrm{keV}$.

Abstract. - The conversion electron spectrum emitted by ${ }^{221} \mathrm{Fr}$ has been studied by means of a $\pi \sqrt{2} \beta$-spectrometer. We determined the multipolarity of the strong transitions. Disagreement has been found between $I \gamma$ and $I e^{-}$for the 73.7 and $99.6 \mathrm{keV}$ radiations, and the assumption of a double level at $99.6 \mathrm{keV}$ has been made.

Les niveaux excités de ${ }^{221} \mathrm{Fr}$, alimentés par l'émission $\alpha$ de ${ }^{225} \mathrm{Ac}$, ont fait l'objet de plusieurs études : K. Valli [1] a étudié les transitions $\gamma$ par une méthode de coïncidences $\alpha-\gamma$ avec jonction $\alpha$ et INa $\gamma$. Wapstra et coll. [2] ont mesuré les spectres $\gamma$ et $e^{-}$en utilisant des détecteurs solides. Dzelepov et coll. [3] ont mesuré 


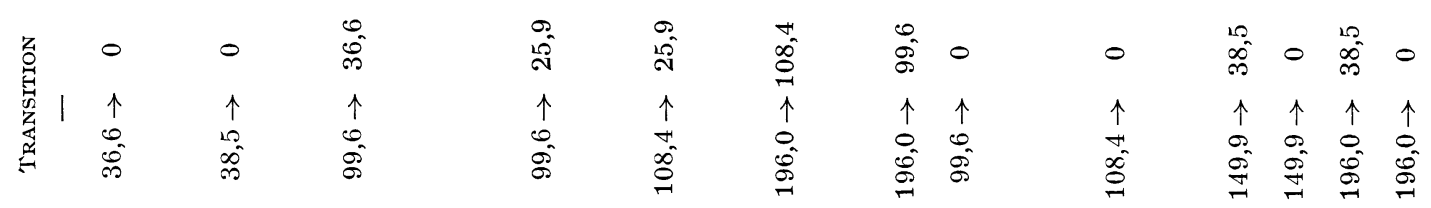

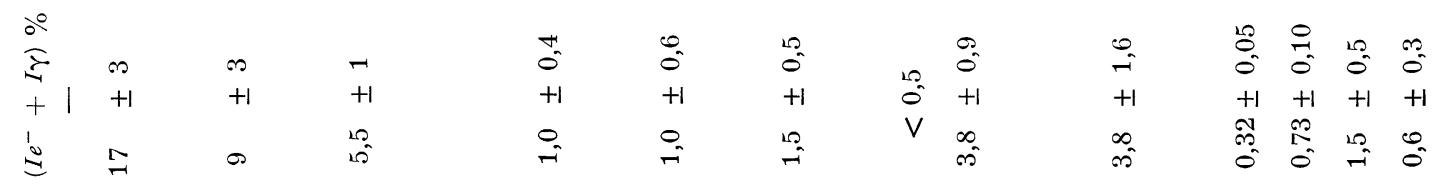

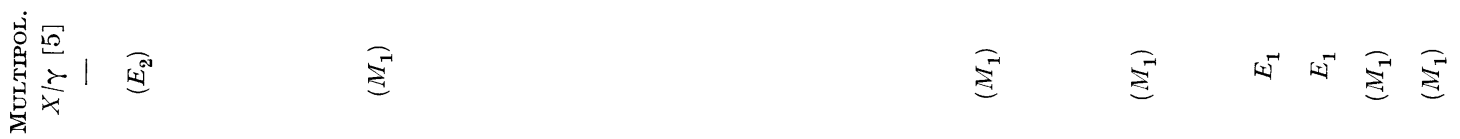

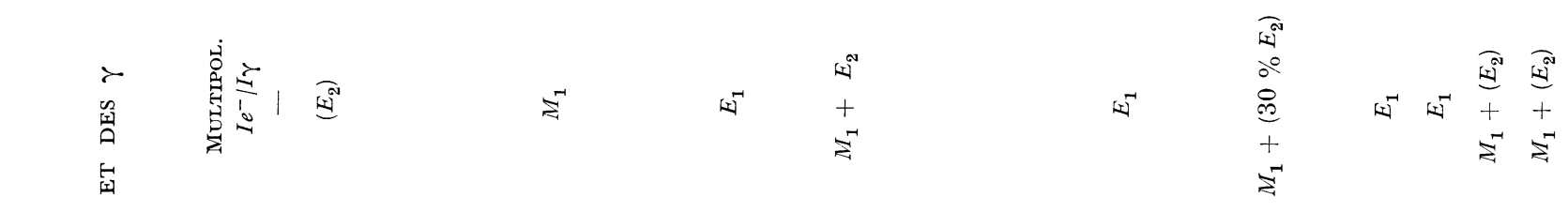

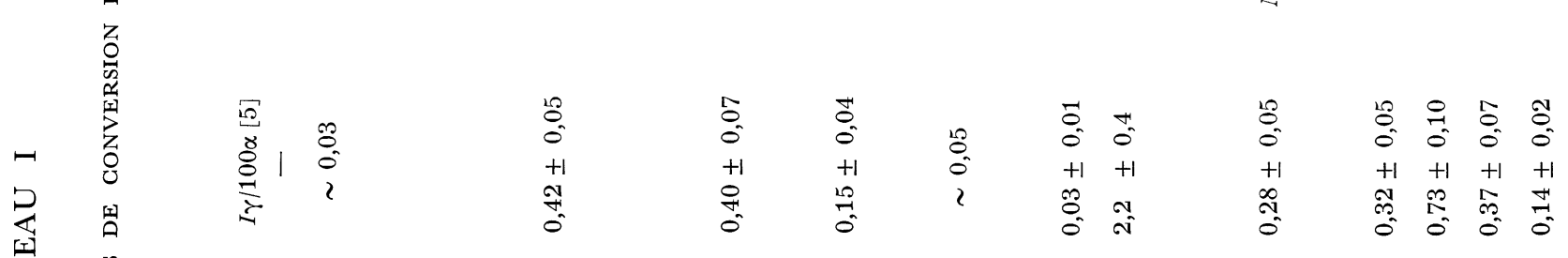

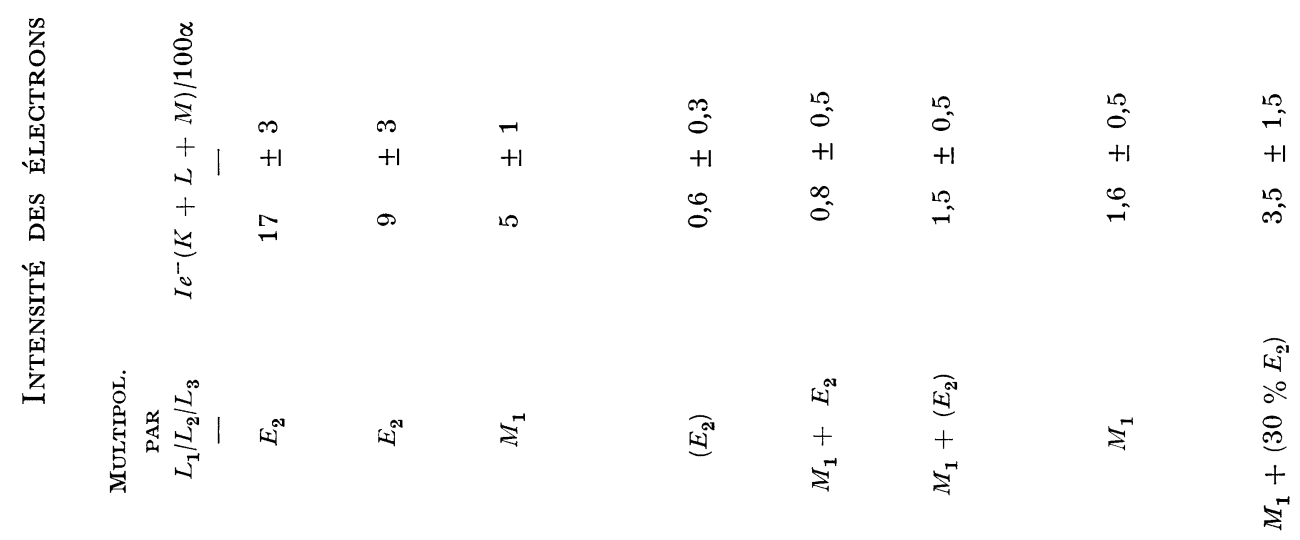

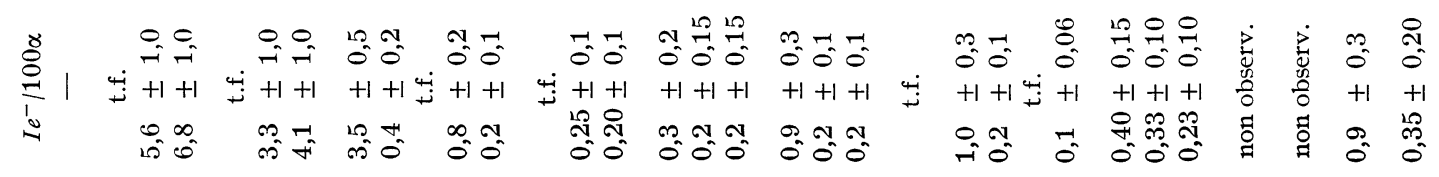

$$
\begin{aligned}
& \text { 甚斯 }
\end{aligned}
$$

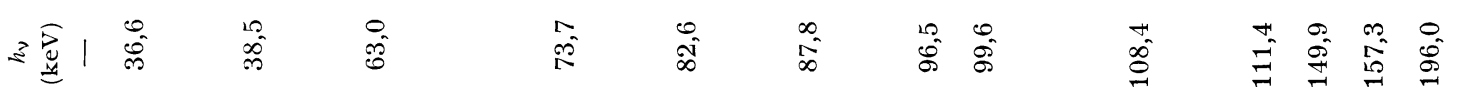


le spectre $\alpha$ magnétique de ${ }^{225} \mathrm{Ac}$. Dans notre Centre, Mme Bastin [4] a également repris l'étude du spectre $\alpha$ magnétique de ${ }^{225} \mathrm{Ac}$ avec le grand spectrographe du C.N.R.S.; elle a pu mettre en évidence deux nouveaux niveaux : 25,4 et 38,2 keV. L'un de nous [5] a étudié les transitions $\gamma$ par une méthode de coïncidences bidimensionnelles entre jonction $\alpha$ et $\mathrm{Ge}(\mathrm{Li}) \gamma$. La plupart des transitions que nous avons observées ont pu être placées dans le schéma déduit de [4], et l'existence de ces deux niveaux a été confirmée. Mais nous avons rencontré des difficultés, dans le cas de coïncidences $\alpha-\gamma$, pour la détermination des multipolarités des transitions déduites seulement à partir des rapports $X / \gamma$. En effet, dans les spectres $\gamma$ de coïncidences, le partage des intensités des $X(L$ ou $K)$, provenant des différentes transitions issues d'un même niveau de départ, est impossible. C'est pourquoi les multipolarités données dans [5] étaient simplement qualitatives, et tenaient compte uniquement des valeurs limites de conversion et du bilan des intensités d'alimentation $\alpha$ et de désexcitation $\gamma$.

L'étude du spectre d'électrons de conversion dans le présent travail nous donne des informations supplémentaires sur ces multipolarités et, en même temps, la possibilité d'interpréter l'ensemble des résultats obtenus.

Appareillage. - Nous avons utilisé le spectromètre $\pi \sqrt{ } \overline{2}$ avec fer, de $50 \mathrm{~cm}$ de rayon, récemment installé dans notre Centre. Le courant de la bobine (stabilisé à $\pm 2 \times 10^{-4}$ ) était mesuré à l'aide d'un voltmètre différentiel, et le détecteur était un compteur Geiger à fenêtre $(20 \times 3 \mathrm{~mm})$ en formvar d'une masse superficielle de $100 \mu \mathrm{g} / \mathrm{cm}^{2}$ environ. L'ensemble du spectrographe et de l'électronique se trouvait dans une salle climatisée à $\pm 1^{\circ} \mathrm{C}$.

Détermination des énergies et des intensités. - On a utilisé comme étalon d'énergie le dépôt actif du thoron $\left({ }^{212} \mathrm{Bi}+{ }^{212} \mathrm{Po}\right)$ et le ${ }^{233} \mathrm{~Pa}$. La correction d'intensité due à l'épaisseur de la fenêtre était de l'ordre de $10 \%$ à $20 \mathrm{keV}$ et négligeable au-delà de $40 \mathrm{keV}$.

En ce qui concerne la détermination de l'angle solide de détection, on a utilisé une source de ${ }^{228} \mathrm{Th}$, en équilibre avec ses descendants, recouverte fortement d'aluminium pour retenir les reculs $\alpha$ successifs. Son activité absolue en $\alpha$ a été étalonnée avec un détecteur solide dans une géométrie bien déterminée. La transition du premier niveau de ${ }^{224} \mathrm{Ra}$ vers le fondamental étant de multipolarité $E_{2}$ pure, l'intensité calculée théoriquement d'après les tables de Rose ou Sliv pour les transitions $E_{2}$, et comparée à celle mesurée expérimentalement, donne directement l'angle solide de détection.

Un contrôle supplémentaire avec la raie $F$ du dépôt actif du thoron, présent dans la même source, nous a donné une valeur semblable :

$$
\Omega=(2,0 \pm 0,2) 10^{-3} \text { (unité } 4 \pi \text { ). }
$$

Source. - L'actinium 225 a été extrait à partir de ${ }^{229} \mathrm{Th}$ fourni par le service de radioéléments du C.E.A. à Saclay, après accumulation de trois mois par intermédiaire de ${ }^{225} \mathrm{Ra}$ :

$$
{ }^{229} \mathrm{Th}(7340 \text { ans }) \stackrel{\alpha}{\rightarrow}{ }^{225} \mathrm{Ra}(15 \mathrm{j}) \stackrel{\beta}{\rightarrow}{ }^{225} \mathrm{Ac}(10 \mathrm{j}) .
$$

La séparation entre $\mathrm{Th}$ et $(\mathrm{Ra}+\mathrm{Ac})$ a été faite par une distillation du chlorure dans un tube de quartz; une deuxième séparation chimique entre $\mathrm{Ra}$ et $\mathrm{Ac}$ a permis d'éliminer ${ }^{225} \mathrm{Ra}$ ainsi que ${ }^{224} \mathrm{Ra}(3,6 \mathrm{j})$ descendant de ${ }^{228} \mathrm{Th}$, qui est présent comme impureté $(\sim 2 \%)$ dans ${ }^{229}$ Th. L'actinium 225 ainsi séparé était volatilisé sous vide sur une tranche d'aluminium de $20 \times 0,8 \mathrm{~mm}$, et avait une activité de $2,5 \mu \mathrm{Ci}$.

Résultats expérimentaux et discussions. - A cause de la faible activité de la source, les mesures ont été limitées aux raies assez intenses correspondant aux transitions des premiers niveaux excités. La bonne résolution du spectromètre permettait de séparer dans la plupart des cas les raies $L_{1}, L_{2}, L_{3}$. La figure 1 montre une partie du spectre et les valeurs expérimentales sont résumées dans le tableau I.

La colonne 4 du tableau I donne la multipolarité de chaque transition déduite uniquement des rapports $L_{1} / L_{2} / L_{3}$. Mais, en utilisant $I e^{-} / 100 \alpha$ (colonne 3 ) et $I \gamma / 100 \alpha$ (colonne 6 ), on peut en déduire le coefficient de conversion absolu, et déterminer ainsi indépendamment une série de multipolarités figurant dans la colonne 7 . La colonne 8 rappelle les attributions antérieures [5].

Dans l'ensemble, les multipolarités s'accordent assez bien, excepté les deux transitions de 73,7 et $99,6 \mathrm{keV}$ pour lesquelles il y a incompatibilité entre les multipolarités déduites de $L_{1} / L_{2} / L_{3}$ et de $I e^{-} / I \gamma$. Sur la figure 1 , on peut constater que les transitions 63,0 et $99,6 \mathrm{keV}$ sont toutes les deux de multipolarité $M_{1}$. A l'aide du coefficient de conversion théorique pour un $M_{1}$, on peut alors calculer l'intensité $\gamma$ correspondante :

$$
\begin{aligned}
& I \gamma(63,0)_{\text {ealc. } M_{\mathrm{\imath}}}=\frac{I e^{-}(L+M+N)}{\alpha_{\text {tot. }}} \\
& \quad=\frac{5 \pm 1}{11,6}=(0,43 \pm 0,1) \%
\end{aligned}
$$

ce qui est à comparer avec :

$$
I_{\gamma}(63,0)_{\text {exp. }}=(0,40 \pm 0,05) \% \text {. }
$$

L'accord entre ces deux valeurs est bon, le rayonnement de $63,0 \mathrm{keV}$ est bien de multipolarité $M_{1}$.

Utilisant le même calcul pour la transition de $99,6 \mathrm{keV}$, on trouve :

$$
I_{\curlyvee}(99,6)_{\text {eale. } M_{1}}=\frac{1,6 \pm 0,5}{3,0}=(0,53 \pm 0,15) \%
$$

alors que :

$$
I_{\curlyvee}(99,6)_{\text {exp. }}=(2,2 \pm 0,4) \% \text {. }
$$




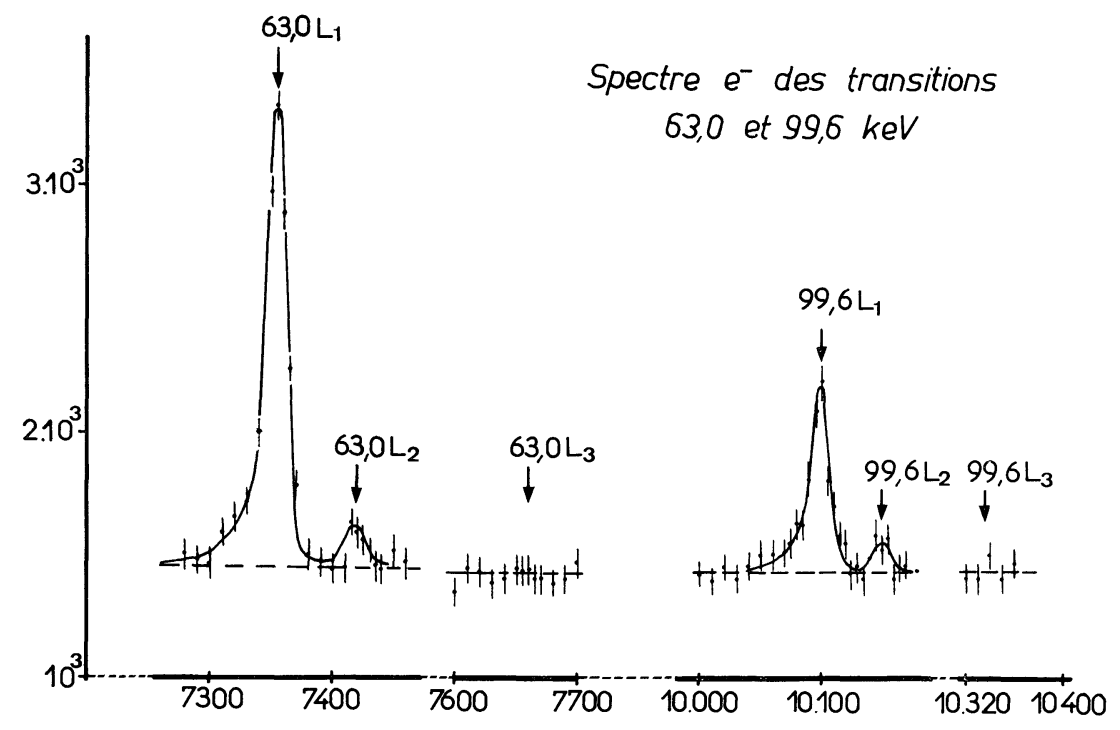

FIG. 1.

L'intensité $\gamma$ expérimentale est quatre fois plus intense que celle évaluée pour un rayonnement $M_{1}$.

Le même phénomène se retrouve pour la transition de $73,7 \mathrm{keV}$ avec $I_{\gamma}$ expérimental, vingt fois trop fort dans l'hypothèse d'un rayonnement $E_{2}$ :

$$
\begin{gathered}
I_{\gamma}(73,7)_{\text {calc. } E_{2}}=\frac{0,6 \pm 0,3}{30}=(0,02 \pm 0,01) \% \\
I_{\gamma}(73,7)_{\exp .}=(0,40 \pm 0,07) \% .
\end{gathered}
$$

Il faut cependant remarquer dans le tableau II que l'alimentation en $\alpha$ du niveau 99,6 correspond bien

\section{TABLEAU II}

Bilan D'Alimentation EN $\alpha$

\begin{tabular}{|c|c|c|c|}
\hline $\begin{array}{l}\text { NIVEAU } \\
-\end{array}$ & $\begin{array}{l}\text { ALIMENT. } \\
\alpha \%[4] \\
\end{array}$ & $\begin{array}{c}\text { DESEXC. \% } \\
\left(e^{-}+\gamma\right) \\
-\end{array}$ & $\begin{array}{c}\text { TRANSITION } \\
-\end{array}$ \\
\hline $6,6+38,5$ & 26,8 & $\begin{aligned} 17 & \pm 3 \\
9 & \pm 3\end{aligned}$ & $\begin{array}{l}36,6 \rightarrow 0 \\
38,5 \rightarrow 0\end{array}$ \\
\hline 99,6 & 10,1 & $\begin{array}{l}5,5 \pm 1,0 \\
1,0 \pm 0,4 \\
3,8 \pm 0,9\end{array}$ & $\begin{array}{l}99,6 \rightarrow 36,6 \\
99,6 \rightarrow 25,9 \\
99,6 \rightarrow 0\end{array}$ \\
\hline 108,4 & 3,4 & $\begin{array}{l}1,0 \pm 0,6 \\
3,8 \pm 1,6\end{array}$ & $\begin{array}{l}108,4 \rightarrow 25,9 \\
108,4 \rightarrow 0\end{array}$ \\
\hline 149,9 & 1,25 & $\begin{array}{l}0,32 \pm 0,05 \\
0,73 \pm 0,10\end{array}$ & $\begin{array}{l}149,9 \rightarrow 38,5 \\
149,9 \rightarrow 0\end{array}$ \\
\hline 196,0 & 4,35 & $\begin{aligned} 1,5 & \pm 0,5 \\
1,5 & \pm 0,5 \\
& <0,5 \\
0,6 & \pm 0,3\end{aligned}$ & $\begin{array}{l}196,0 \rightarrow 108,4 \\
196,0 \rightarrow 38,5 \\
196,0 \rightarrow 99,6 \\
196,0 \rightarrow 0\end{array}$ \\
\hline
\end{tabular}
Et DÉSeXcitation $\left(e^{-}+\gamma\right)$ à la somme des désexcitations $e^{-}+\gamma$ des transitions de $63,0,73,7$ et $99,6 \mathrm{keV}$. Ceci confirme la validité de nos valeurs expérimentales.

Le désaccord sur les intensités $\gamma$ calculées et expérimentales pour les deux transitions de 73,7 et $99,6 \mathrm{keV}$ nous a conduits à examiner les hypothèses suivantes :

1) Il s'agit d'une anomalie de conversion interne, mais il n'a jamais été mis en évidence jusqu'ici des conversions aussi anormales pour des rayonnements $M_{1}$ ou $E_{2}$

2) Le niveau 99,6 pourrait être double, les rapports $\gamma / e^{-}$étant très différents pour les rayonnements issus de ces deux niveaux.

Il a donc été très important de remesurer avec précision $E \gamma$ et $E e^{-}$pour essayer de montrer éventuellement qu'il s'agit d'un doublet.

Une détermination plus précise en énergie de la raie $L_{1}$ de $99,6 \mathrm{keV}$ a été faite en utilisant comme étalon, dans des conditions de parfaite reproductibilité, la raie $L_{1}$ d'une transition bien connue de $(103,86 \pm 0,02) \mathrm{keV}$ dans la désintégration $\beta \mathrm{du}$ ${ }^{233} \mathrm{~Pa}$ [6], les deux raies étant très proches en énergie.

La différence trouvée entre ces deux raies est :

$$
\Delta E e^{-}=1,20 \pm 0,10 \mathrm{keV} .
$$

L'erreur indiquée ici provenait surtout de la faible statistique sur la raie $L_{1} \mathrm{du} 99,6 \mathrm{keV}$.

En tenant compte de l'énergie de liaison $L_{1}$, on trouve :

$$
h_{\nu\left(e^{-}\right)}=99,56 \pm 0,10 \mathrm{keV} .
$$

Une mesure des énergies $\gamma$ a été faite également, en prenant comme étalons les $\gamma$ de ${ }^{241} \mathrm{Am}: 99,00$ $\pm 0,04 \mathrm{keV}$ [7] et $102,96 \pm 0,04 \mathrm{keV}$ [8]. 


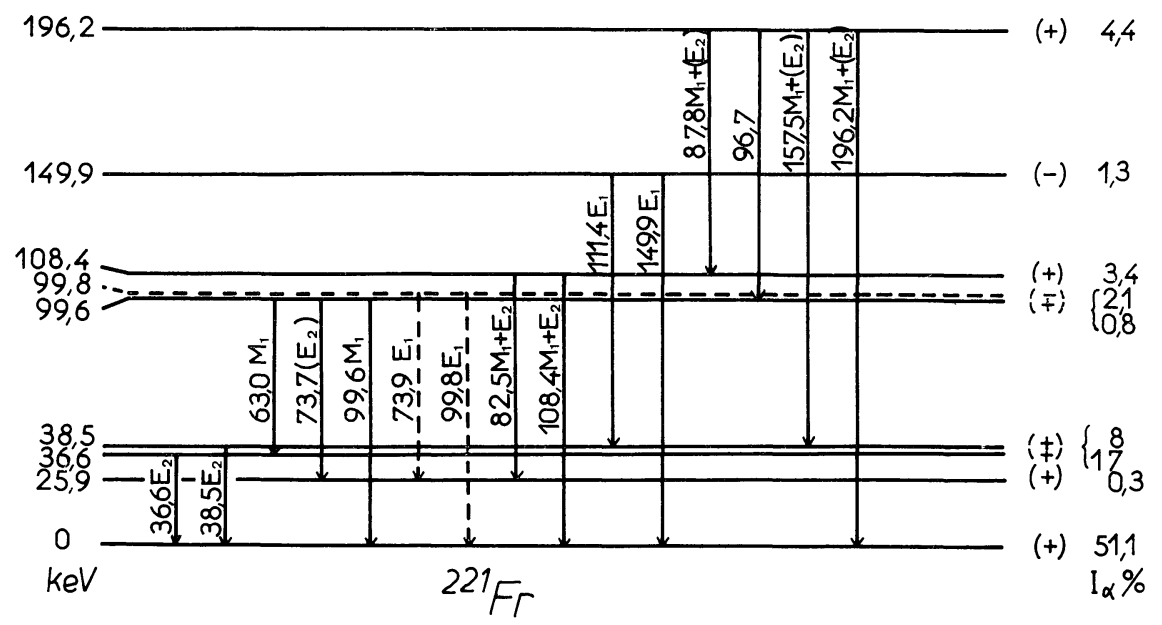

FIG. 2.

L'énergie de transition par voie $\gamma$ ainsi trouvée est :

$$
h_{\nu(\gamma)}=99,80 \pm 0,10 \mathrm{keV} \text {. }
$$

La différence de l'énergie de transition ainsi trouvée : $0,24 \pm 0,14 \mathrm{keV}$, n'est pas très concluante.

Nous avons néanmoins utilisé de préférence la deuxième hypothèse mentionnée ci-dessus pour proposer, en ce qui concerne les premiers niveaux, le schéma de ${ }^{221} \mathrm{Fr}$ reproduit dans la figure 2 et basé sur l'ensemble des données obtenues par spectrographie $\alpha$, $\gamma$ et de conversion.

Le niveau supplémentaire à $99,8 \mathrm{keV}$, introduit ici par suite des considérations sur l'interprétation des intensités, devrait être de parité opposée à celui de $99,6 \mathrm{keV}$. L'intensité $\alpha$ alimentant ces deux niveaux est évaluée sur le bilan de désexcitation $e^{-}+\gamma$.

Nous remercions beaucoup M. Walen et Mme Bastin pour la préparation de la source de ${ }^{225} \mathrm{Ac}$ à partir $\mathrm{du}{ }^{229} \mathrm{Th}$, ainsi que M. Sant'Ana Dionisio qui a bien voulu mettre à notre disposition le spectromètre $\pi \sqrt{2}$.

(*) Ce travail fait partie de la thèse de M. Ching-Fan Leang, enregistrée au C.N.R.S. sous le no A.O. 3075 .

\section{BIBLIOGRAPHIE}

[1] Valli (K.), Ann. Acad. Sc. Fennica, Physica, 1964, 6, série A, 165.

[2] Nuclear Data Sheet, Academic Press, novembre 1966 , section $B, 1,5$.

[3] Dzelepov (B. S.), Ivanov (R. B.), Moskvin (L. N.) et Rodionov (B. F.), Izvestia, Akad. Nauk. SSSR, 1966, 30, 1322.

[4] Bastin (Mme G.), C. R. Acad. Sc. Paris, 1967, 265, série $\mathrm{B}, 863$.
[5] LEang (Chin-Fan), C. R. Acad. Sc. Paris, 1967, 265 série $B, 417$.

[6] Albridge (R. G.), Hollander (J. M.), Gallagher (C. J.) et Hamition (J. H.), Nucl. Phys., 1961, 27, 529 .

[7] Wolfson (J. L.) et Park (J. H.), Can. J. Phys., 1964, 42, 1387.

[8] Yamazaki (T.) et Hollander (J. M.), Nucl. Phys., 1966, 84, 505 . 